in: Nonlinear Physics of Complex Systems -- Current Status and Future Trends, J. Parisi, S.

C. Müller, and W. Zimmermann, eds.

Lecture Notes in Physics, vol. 476, pp. 294-308, Springer, Berlin (1996)

\title{
Brownian Rectifiers: How to Convert Brownian Motion into Directed Transport
}

\author{
Peter Hänggi and Roland Bartussek \\ Institut für Physik, Universität Augsburg \\ Memminger Str. 6, D-86135 Augsburg
}

PACS: 05.40.+j, 82.20.Mj, 87.10.+e, 74.20.De

\section{Introduction}

It is generally appreciated that - in accordance with the second law of thermodynamics - usable work cannot be extracted if only equilibrium fluctuations act. The physical mechanism that prevents to pullout work from equilibrium fluctuations is tutorially discussed in Feynman, Leighton, and Sands [1]: In doing so they use a molecular gadget composed of a ratchet, a pawl and a set of vanes, see Fig. 1. Actually this Brownian machinery goes

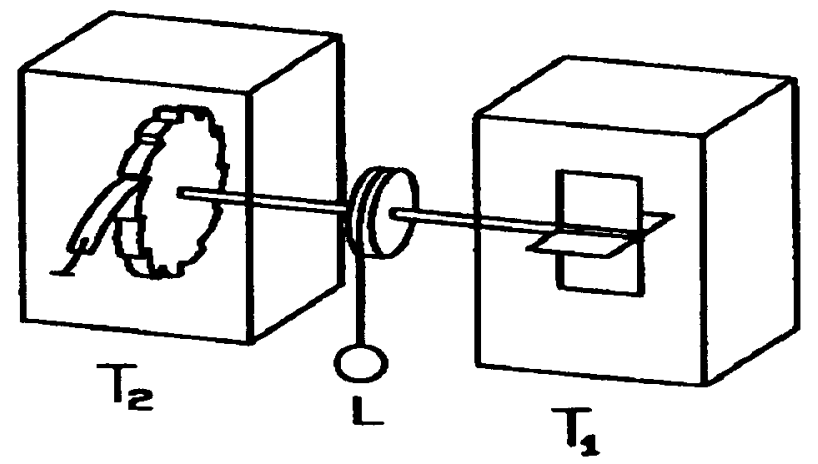

Fig. 1. The 'ratchet and pawl' machine: Two heat reservoirs are connected by an axle that is attached to a ratchet in heat bath $T_{2}$ and to vanes in heat bath $T_{1}$. Due to the bombardments of gas molecules in heat bath $T_{1}$ on the vanes the ratchet is forced in both directions, with the backward motion being blocked on average by the pawl. When $T_{1}=T_{2}$ no net motion occurs, in agreement with the second law of thermodynamics. When $T_{1}>T_{2}$ a small weight $L$ can be lifted.

back to a stimulating presentation put forward by Marian von Smoluchowski [2] during the heydays of early Brownian motion. In his discussion [2], which 
up to present days provides charitable reading, Smoluchowski shows that in absence of a superintelligent creature, i.e., a Maxwell demon, no net current will emerge. In presence of nonequilibrium forces the situation changes drastically: Now a ratchet potential $V_{R}(x)$, that is a periodic structure which lacks the reflection symmetry, can rectify unbiased nonequilibrium fluctuations into a fluctuation induced directed current. Put differently, by a ratchet system we have in mind a gadget that is able to move particles with a nonzero velocity in absence of macroscopic bias forces such as field gradients of electrical, thermal or chemical potential origin, etc.. Strictly speaking, we define a ratchet system as a system that is able to transport particles in a periodic structure with nonzero macroscopic velocity although on average no macroscopic force is acting. Such nonequilibrium ratchet systems recently gained much interest [3] in view of their role in describing the physical aspects that are involved in the working principle of motor proteins (i.e., molecular motors) and, likewise, their potential for novel technological applications on nano- and microscales. In the following we shall address several possibilities of obtaining directed motion of particles in periodic structures.

\section{Current as a Consequence of State-Dependent Noise}

The application of a periodic, but nonuniform heat-cool mechanism (i.e., the blow torch effect [4]) that is not 'in phase' $[5,6]$ with a reflection-symmetric, periodic potential will - upon application (!) of periodic boundary conditions - yield a finite current $[5,6]$. The nonuniform temperature (or diffusion) is rooted in a multiplicative, periodic white Gaussian noise. For overdamped, onedimensional motion this multiplicative noise can readily be transformed into additive noise [6]. The corresponding effective potential $\Psi(x)$ is then no longer periodic but exhibits an average bias. Put differently, taken over the length $L$ of the periodic structure, the potential difference $\Psi(x=L)$ $\Psi(x=0) \equiv \Delta \Psi$ is no longer zero, but enters the exponential behavior of the probability current $[5,6]$. A variant of this 'blow torch effect' induced current mechanism is obtained if we start out with a periodic, but asymmetric ratchet potential, $V_{R}(x)=V_{R}(x+L)$, and drive the system by multiplicative thermal noise that now is 'in phase' with the force $-V_{R}^{\prime}(x)[7]$. Here, the asymmetry of the heat-cool mechanism over the period $L$ of the ratchet structure mimics the 'out-of phase' blow torch effect in a symmetric potential. We note, that in all these cases the periodic structure is subjected to a macroscopic thermal gradient. Hence, we do not classify such systems as ratchet systems in the strict sense. From a physics point of view, these systems are related closely to the ratchet and pawl gadgets that perform usable work only in presence of a temperature gradient $T_{1} \neq T_{2}$, see in this context also the insightful discussion in Ref. [8].

Let us next address a ratchet system that in spirit is closely related to the above systems driven by state dependent noise. In this ratchet a nonzero 
current results from the modulation of an asymmetric potential profile. To be precise, we consider the transport of overdamped particles in a ratchet potential $V_{R}(x)$ whose dynamics is governed by the Langevin equation

$$
\dot{x}=-\zeta(t) V_{R}^{\prime}(x)+\sqrt{2 D} \xi(t)
$$

Here $\xi(t)$ denotes additive Gaussian white noise (e.g., a thermal Nyquist noise source) of zero mean and correlation $\langle\xi(t) \xi(s)\rangle=\delta(t-s)$. The nonequilibrium forcing $\zeta(t)$ that rules the time dependent change of the potential profile is either a periodic, deterministic modulation with period $\mathcal{T}$, i.e., $\zeta(t) \rightarrow F(t)$ $=F(t+\mathcal{T})$, or a stochastic, non-white process $\zeta(t)$. Of particular interest is the case with $\zeta(t)$ or $F(t)$ assuming the values 0 and 1 only. This corresponds to a situation where the periodic potential $V_{R}(x)$ is switched $\mathbf{O N}$ and OFF, cf. Fig. 2. In the following we shall classify this situation as a

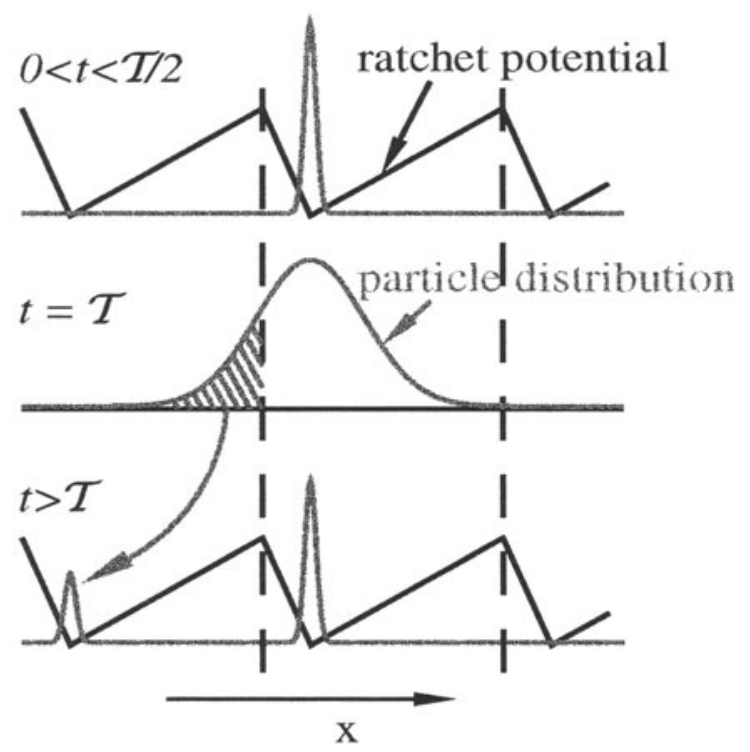

Fig. 2. The noise induced transport mechanism in a ratchet potential that is periodically (period $\mathcal{T}$ ) switched ON and OFF: A particle distribution which is initially located in a minimum of the potential will spread symmetrically by diffusion while the potential is switched OFF. When the potential is switched ON again, a net part of the distribution will settle in the minimum located towards the left. Hence, on average a particle current flows to the left.

flashing ratchet mechanism [9-12], see below. The physical mechanism that governs the transport is indicated with Fig. 2. Note that the particles on average are transported into the direction of larger average force $\left|V_{R}^{\prime}(x)\right|$, i.e., 
into a direction opposite to the polarity of the ratchet $V_{R}(x)$. This polarity is positive if the average force $\left|V_{R}^{\prime}(x)\right|$ is smaller in forward direction. In Fig. 2, this polarity is positive; hence the noise induced current is towards the left.

As indicated with $(1)$, the force $-\zeta(t) V_{R}^{\prime}(x)$ formally mimics a nonuniform source of non-white diffusion. The conditioned expectation of the force $-\left\langle\zeta(t) V_{R}^{\prime}(x(t)) \mid x(t)=x\right\rangle$ is not identically vanishing. Nevertheless, on average, $\int_{0}^{L}\left\langle\zeta(t) V_{R}^{\prime}(x(t)) \mid x\right\rangle d x=0$, i.e., the noise-induced force vanishes on average over the period $L$ of the periodic structure. In this sense, this ratchet mechanism is related from a physics point of view to the asymmetric heatand-cool effect that characterizes the current induced by nonuniform, periodic diffusion [5]. A distinct feature of the flashing ratchets in (1) should be pointed out however: For $\zeta(t)$ approaching white noise (either Gaussian white [6] or Poissonian white [13]) the noise induced current is vanishing. Thus, with white noise forces a current results only in presence of an additional nonvanishing drift field [as given by the force of a periodic potential $V(x)=V(x+L)]$. Put differently, nonuniform, but periodic white noise of the form $\left[-\zeta(t) V_{R}^{\prime}(x)+\sqrt{2 D} \xi(t)\right]$ alone does not result in a finite current! Also, with $D$ set 0 , colored nonuniform noise in (1) alone yields no net current.

The flashing ratchet mechanism very recently has been experimentally tested in a ratchet potential $V_{R}(x)$ created with the help of a microelectrode system (Christmas-tree electrodes) [14] as well as in a potential created by optical means [15].

Before we proceed, let us characterize the variants among the ratchet systems in more definite terms. Among ratchets that move particles forward noisily we can distinguish the following types:

i) A first class of ratchet systems is given by the above discussed Flashing Ratchets. Therein, the particles experience a fluctuating energy profile [9$12,14,15]$. An equivalent ratchet mechanism occurs for particles occupying discrete states with transitions among them that do not obey detailed balance, see in Refs. $[9,10]$.

ii) Another class are particles moving in an asymmetric periodic potential subjected solely to spatially uniform forces of (temporal or statistical) zero average. We shall term these systems Changing-Force Ratchets. These systems again can be divided into three subclasses, namely:

(a) Rocking Ratchets, in which the particles experience the action of spatially uniform, time-periodic deterministic forces, $F(t)=F(t+\mathcal{T})$ [16-20,29,30].

(b) Diffusion Ratchets, when driven by a spatially uniform, time-periodic diffusion coefficient $D(t)=D(t+\mathcal{T})[21]$.

(c) Correlation Ratchets, when driven by spatially uniform stochastic forces $\zeta(t)$ of zero average [16,22-28]. 


\section{Directed Transport Induced by Rocking}

Instead of a fluctuating energy profile we consider overdamped motion subject to both, a periodic force-modulation of zero temporal average and thermal white noise, i.e., [16-19]

$$
\dot{x}=-V_{R}^{\prime}(x)+A \sin (\Omega t)+\sqrt{2 D} \xi(t) .
$$

Inspecting the ratchet dynamics in (2) in the adiabatic limit, i.e., $\Omega \rightarrow 0$,

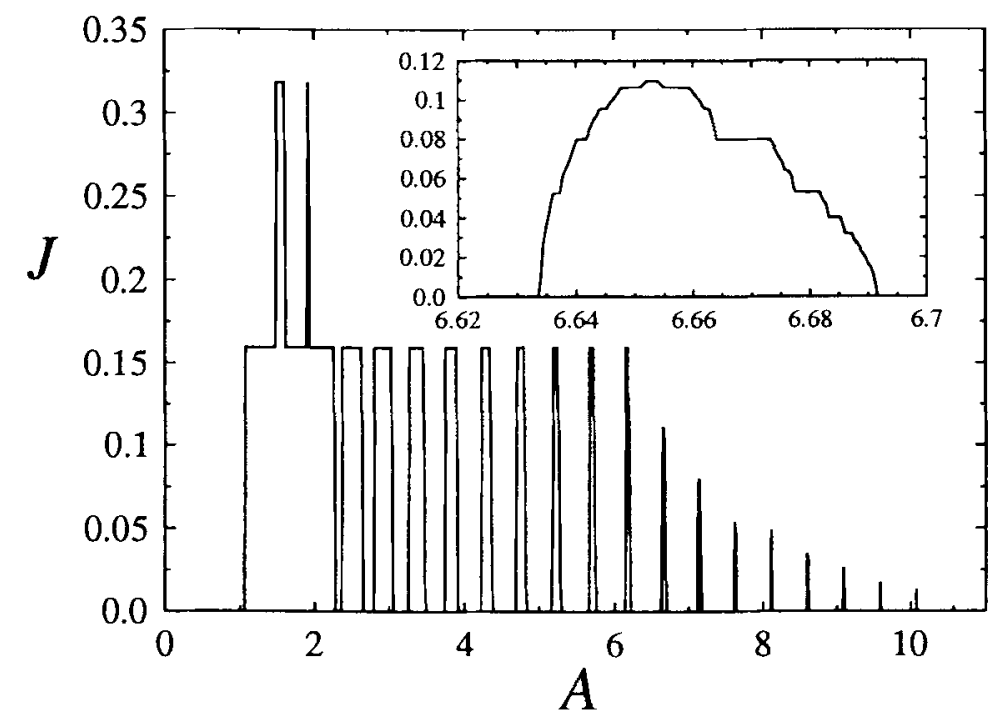

Fig. 3. The average current in a periodically rocked ratchet, eq. (2), without noise $(D=0)$ is depicted versus the amplitude of driving $A$ for a fixed frequency of driving $\Omega=1$. The ratchet potential is chosen as $V_{R}(x)=-[\sin (2 \pi x)+0.25 \sin (4 \pi x)] / 2 \pi$. Note that the current is zero below a lower threshold value for $A$; interestingly enough the current also locks into zero for large driving amplitudes. The locking mechanism for the plateau widths is governed by a devil's staircase (note the inset).

we notice that for a force strength $A$ that exceeds the maximal forward force but not the maximal backward force of $-V_{R}^{\prime}(x)$ (see Fig. 2 for a forward ratchet) the particles are on average pushed into the forward direction. Thus, this mechanism works even in absence of thermal noise $\xi(t)$. Note that the direction of the current in a changing force ratchet is opposite to the direction in a flashing ratchet - assuming here no current reversal phenomena as a function of parameters entering (2), see below. In terms of the spatially 
periodic probability current $J$, the particle current $\langle\dot{x}\rangle$ is given by

$$
\langle\dot{x}\rangle=L J .
$$

For a deterministically rocked ratchet with $D=0$ the space and time averaged probability current $J$ is depicted in Fig. 3 versus the amplitude strength $A$. Even in this most simple deterministic situation the current behavior is rather complex: One finds that at fixed frequency $\Omega$ the current-plateau values exhibit a locking mechanism that can be theoretically described by a devil's staircase [17-19]. This feature is most conveniently studied within a stroboscopic-like description of the continuous-time dynamics [19].

In presence of noise, the study of the time-dependent Langevin equation in (2) presents a challenge. With the driving being in the nonadiabatic regime the corresponding time-periodic Fokker-Planck equation must be studied by means of a Floquet analysis [18]. In Fig. 4 we depict the noise-induced cur-

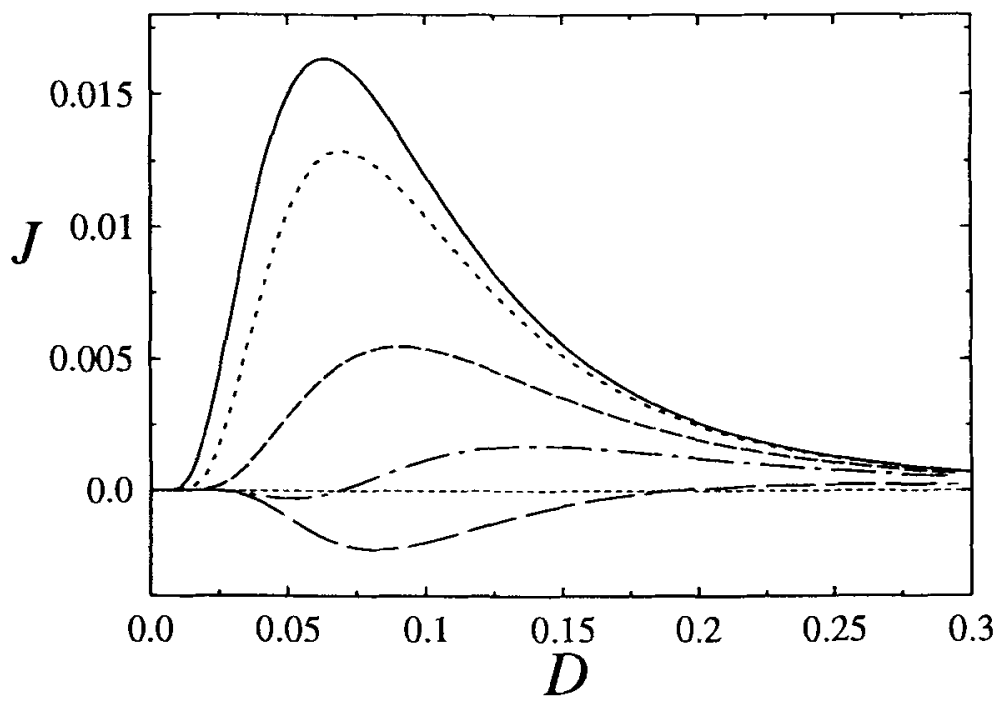

Fig. 4. The addition of thermal noise of strength $D$ to the rocking ratchet described in eq. (2) and Fig.3 leads to a current reversal as a function of $D$ at moderate-to-large driving frequencies $\Omega$ : For a fixed amplitude of driving $A=1$ the driving frequencies are $\Omega=0.01,1,2.5,4,7$ (from top to bottom). Figure taken from Ref.[18].

rent versus the noise intensity $D$. Worthy to point out from Fig. 4 is the bell-shaped, resonance like feature of the probability current $J$ versus $D$. Moreover, we find the phenomenon of a current reversal [18] that occurs for nonadiabatic driving frequencies $\Omega$. 
The mean velocity $\langle\dot{x}\rangle$ of the particle position in the adiabatic approximation $(\Omega \rightarrow 0)$ is explicitly given by [18]:

$$
\begin{aligned}
\langle\dot{x}\rangle= & L J \\
=\frac{L D}{2 \pi / \Omega} \int_{0}^{2 \pi / \Omega} d t\{ & \frac{1}{1-\exp [\Phi(L, t)]} \int_{0}^{L} d x \int_{0}^{L} d y \exp [\Phi(y, t)-\Phi(x, t)] \\
& \left.\quad-\int_{0}^{L} d x \int_{0}^{x} d y \exp [\Phi(y, t)-\Phi(x, t)]\right\}^{-1}
\end{aligned}
$$

where $\Phi(x, t)=\left[V_{R}(x)-x A \sin (\Omega t)\right] / D$. This approximation coincides within line-thickness with the exact Floquet-theory result for the smallest frequency $\Omega=0.01$ in Fig. 4 (solid line).

The effects of inertia and/or weak friction are also intriguing [20]: In absence of thermal noise, the characteristic chaotic motion is sufficient to induce a directed current which undergoes multiple current reversals versus the driving amplitude $A$. The chaotic transport itself exhibits a Gaussian asymptotic scaling behavior.

Another ratchet type that is related to the rocking ratchet in Eq.(2), is obtained if one substitutes the external coherent driving by an oscillating temperature (diffusion ratchet) [21]. The overdamped transport with a rocking diffusion is governed by the Langevin equation

$$
\dot{x}=-V_{R}^{\prime}(x)+\sqrt{2 D} \xi(t)[1+A \sin (\Omega t)] .
$$

This ratchet gadget tends to resist to carry a finite current in both asymptotic limits of fast and slow driving: The current starts only proportional to $\Omega^{2}$ as $\Omega \rightarrow 0$ (zero net current in the adiabatic limit), and vanishes again proportional to $\Omega^{-2}$ as $\Omega \rightarrow \infty$, see in Fig. 5 .

\section{Rectification of Noise with Correlation Ratchets}

The nonequilibrium forces most commonly used in rectifying Brownian motion into directed transport are nonthermal, stochastic forces $\zeta(t)$. The application of a spatially uniform force $\zeta(t)$ brings the ratchet dynamics away from thermal equilibrium $[16,22-28]$. As a consequence, the stochastic dynamics no longer obeys the principle of detailed balance which is the hallmark for any equilibrium system.

Let us investigate here in greater detail the necessary ingredients for noise induced transport along a periodic structure. First, a finite current can emerge only if, on average, noise activated forward and backward transitions do not balance each other. The key ingredient for noise rectification thus is an asymmetry between forward and backward hopping rates. For this to be the case detailed balance symmetry must be broken. This occurs either via the 


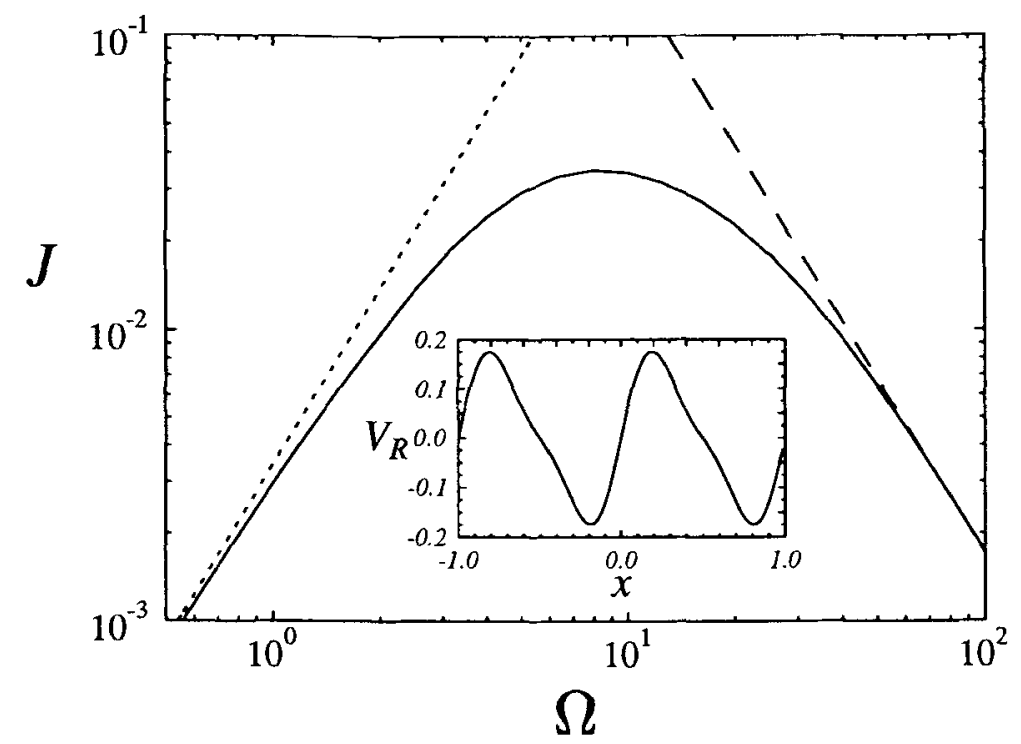

Fig. 5. Probability current $J$ versus oscillation frequency $\Omega$ for the diffusion ratchet (5) at the parameter values $D=0.1$ and $A=0.7$. The solid line shows numerical results for a Floquet analysis of the time periodic Fokker-Planck equation corresponding to (5). The dashed line is the theoretical large- $\Omega$ asymptotics (see in Ref.[21]). The dotted straight line of slope 2 corresponds to the $\Omega^{2}$-asymptotics from a second order adiabatic theory. The used ratchet potential, plotted in the inset, is the negative of the potential used in Fig.3; thus, the direction of current in a diffusion ratchet is opposite to the direction in a rocking ratchet.

application of nonthermal forces $\zeta(t)$, or time dependent deterministic periodic forcing as discussed above. Taken alone this is, however, not sufficient. A counterexample is given with symmetric, correlated (i.e. colored) noise (e.g., Gaussian colored noise) driven transport in a reflection-symmetric potential $V(x)$. Then, on average, no net current results. To obtain a finite current the dynamics in addition must experience a source of ASYMMETRY. An obvious source of asymmetry comes in with the asymmetry of the ratchet potential $V_{R}(x)$. Hence, a finite current in a correlation ratchet $V_{R}(x)$ results already whenever the nonthermal fluctuating forces $\zeta(t)$ are symmetric. Put differently, all noise sources of zero average $\langle\zeta(t)\rangle=0$ that possess zero, odd-numbered cumulant averages $C_{2 n+1}=\left\langle\zeta\left(t_{1}\right) \ldots \zeta\left(t_{2 n}\right)\right\rangle_{c}=0, n=1,2, \ldots$, will generally support a nonvanishing macroscopic velocity only in a ratchet potential $V_{R}(x)$. In presence of an asymmetry for the nonthermal, additive noise source $\zeta(t)$, namely in presence of odd-numbered noise-cumulants obeying $C_{2 n+1} \neq 0$, a reflection-symmetric periodic structure $V(x)$ is sufficient to support a current. 
For overdamped motion, the dynamics for a correlation ratchet is governed by a nonthermal, in general colored noise driven Langevin equation in a periodic potential, i.e.,

$$
\dot{x}=-V^{\prime}(x)+\zeta(t)+\sqrt{2 D} \xi(t)
$$

White shot noise $\zeta(t)$ of zero average possesses $\delta$-correlated odd higher order cumulants [13]

$$
C_{2 n+1}=(2 n+1) ! \lambda B^{2 n+1} \delta\left(t_{1}-t_{2}\right) \times \ldots \times \delta\left(t_{2 n}-t_{2 n+1}\right), \quad n=1,2, \ldots
$$

Hence, a reflection symmetric potential $V(x)$ is sufficient to induce a directed, nonzero average particle current [25].

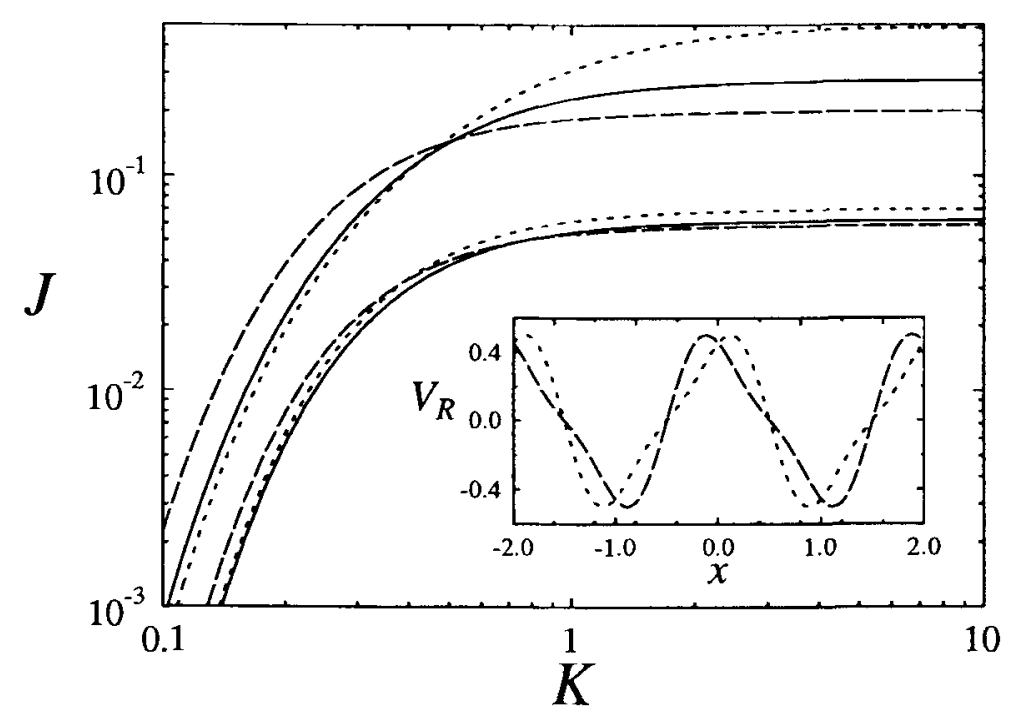

Fig. 6. The probability current $J=\langle\dot{x}\rangle / L$ for a white shot noise driven correlation ratchet [25] is shown versus the noise strength $K$ for different shapes of the potential: Solid lines are for the symmetric potential $V(x)=(1 / 2) \cos (\pi x)$, dashed and dotted lines for the ratchet potentials $V_{R}(x)=0.454[\cos (\pi x) \pm 0.25 \sin (2 \pi x)]$, the latter potentials are depicted in the inset. The parameter $\lambda B$ is 2.5 in the upper curves and $\lambda B=10$ for the lower ones, see $(6),(7)$.

Interestingly enough, the exact average particle velocity in this case can be

\footnotetext{
${ }^{1}$ Likewise, a current emerges in a symmetric potential with a rocking mechanism $F(t)=F(t+\mathcal{T})$, that is periodic with zero temporal average and which is not reflection-symmetric within a period $\mathcal{T}[17]$.
} 
evaluated in analytical closed form if we set the thermal noise intensity $D$ equal to zero. From the corresponding master equation the current explicitly reads [25]

$$
\langle\dot{x}\rangle=L \frac{1-\exp [\Psi(L)]}{\int_{0}^{L} d x D_{e f f}^{-1}(x) \exp [-\Psi(x)] \int_{x}^{x+L} d y \exp [\Psi(y)]} .
$$

Here, the effective diffusion $D_{\text {eff }}$ and the generalized potential $\Psi(x)$ are defined by

$$
D_{e f f}(x)=K\left[1+\frac{V^{\prime}(x)}{\lambda B}\right], \quad \Psi(x)=\int_{0}^{x} d y \frac{V^{\prime}(y)}{D_{e f f}(y)},
$$

with $K \equiv \lambda B^{2}$.

For the reflection symmetric potential $V(x)=\cos (\pi x) / 2$ the probability current $J=\langle\dot{x}\rangle / L=\langle\dot{x}\rangle / 2$ is depicted in Fig. 6 with the solid line. Therein, the current is also compared with white shot noise driven ratchets of opposite polarity. A remarkable result is that at small-to-moderate noise intensity $K$ the negative polarity ratchet yields a current that is larger than the current in a reflection-symmetric potential, and also larger than the current in a forward ratchet. In absence of white Gaussian noise, a similar analytical closed form study for the ratchet current is possible also for $\zeta(t)$ being symmetric dichotomic noise [22] or asymmetric dichotomic noise of zero average [27].

\section{Current Reversals}

We already noted for the rocking ratchet that naive, adiabatic arguments predicting the direction of current in changing-force ratchets subject to symmetric forcing do not necessarily give the correct sign for nonadiabatic forcing. As depicted with Fig. 4, the nonadiabatic driving regime exhibits at smallto-moderate noise intensity $D$ a reversal of current direction. In correlation ratchets, i.e., $F(t) \rightarrow \zeta(t)$, we hence expect similar results, namely that the current direction cannot readily be predicted a priori. Indeed, the study of current-reversal phenomena has given rise to a research activity on its own $[18,22-24,26,28,29]$. For the simplest, nontrivial case of a correlation ratchet driven by exponentially correlated Gaussian noise [26], i.e.,

$$
\dot{x}=-V_{R}^{\prime}(x)+\zeta(t)+\sqrt{2 D} \xi(t),
$$

with

$$
\langle\zeta(t) \zeta(s)\rangle=\frac{Q}{\tau} \exp (-|t-s| / \tau),
$$

we depict with Fig. 7 the resulting current reversal as a function of noise color $\tau$ for a ratchet potential composed of three Fourier modes [26]. Note in Fig. 7 that the current vanishes at a certain value of noise color $\tau$. An interesting feature related to the phenomenon of current reversal is its suppression with increasing cooperativity, as given by the increase of particle density of finitesized overdamped particles in a ratchet system [29]. 


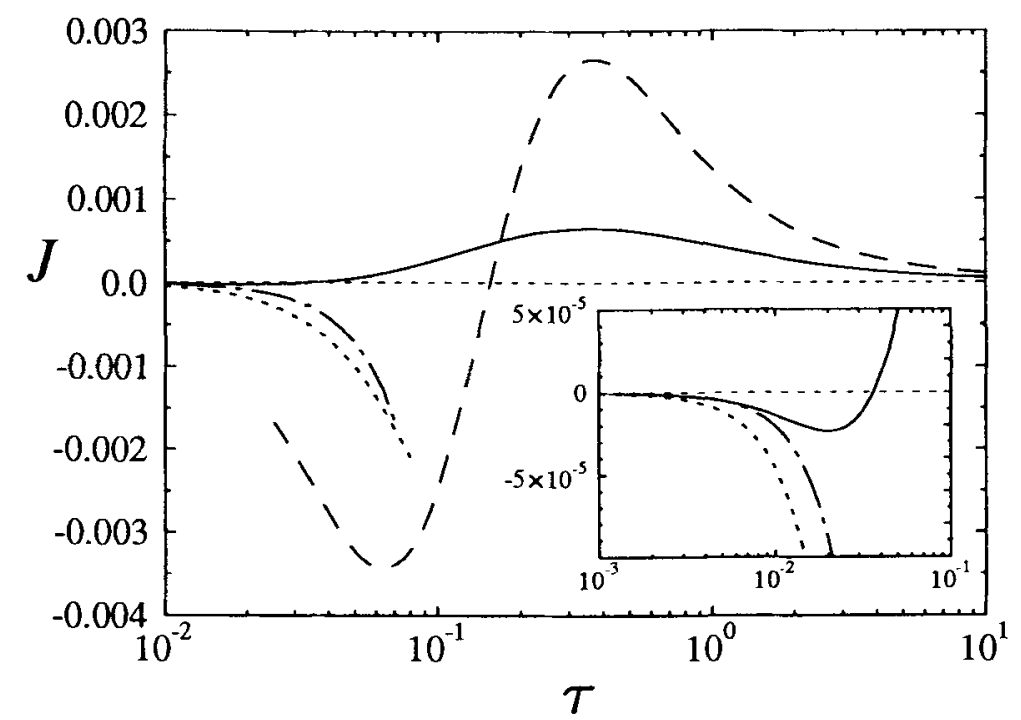

Fig. 7. The current reversal phenomenon in a correlation ratchet, eqs.(10),(11): The probability current $J$ is depicted for a white noise intensity $D=0.05$ and colored noise intensity $Q=0.025$ versus the correlation time $\tau$ of Ornstein-Uhlenbeck noise $\zeta(t)$. The ratchet potential is composed of three Fourier modes: $V_{R}(x)=-\{\sin (2 \pi x)+0.2 \sin [4 \pi(x-0.45)]$ $+0.1 \sin [6 \pi(x-0.45)]\} / 2 \pi$. Numerical results (solid) are compared to generalized unified colored noise approximation (dashed-dotted), small- $\tau$ theory (dotted), and 'small- $\gamma$ ' (dashed) theory (see in Ref.[26]). The inset gives a magnification of the behavior at small $\tau$.

\section{Applications Using Noise Rectification}

Although rectification processes are known for a long time, we argue that these can assume interesting characteristics within the periodic ratchet structures discussed here. The fact that directed noise induced transport can occur in periodic structures may lead to a new generation of separation and/or pumping techniques. Most separation techniques known to date are based on the use of an externally applied static load force $F_{0}$. Then the particles generically move into the direction of the load. New characteristics appear when we use stochastic forces $\zeta(t)$, or alternating deterministic forces $F(t)$. As demonstrated with the ratchet mechanism, a directed nonzero current then can emerge even in absence of a load $F_{0}$. What matters mostly for useful technological applications of ratchets is the amount of work that a particle performs in presence of the additional load $F_{0}$. Thus, of particular interest 
is the behavior of current versus external load. In Fig. 8 we depict the current versus load curve for the Gaussian correlated noise driven correlation ratchet, eqs.(10),(11). Here, the no-load current is given by the intercept on the vertical axis. The stopping force $F_{\text {stop }}$ is the load value where the current assumes zero against the static load $F_{\text {stop }}$ and is given by the intercept with the horizontal axis. Because of a nonzero no-load current the ratchet mechanism yields a novel effect: Particles placed into a periodic potential profile can be moved uphill against an external bias until the stopping force is reached! We notice that the rectification of current is not exponential-like but rather mimics a linear behavior versus $F_{0}$ with a slope that is different within the linear response regime (small $F_{0}$ ) as compared to the large load regime.

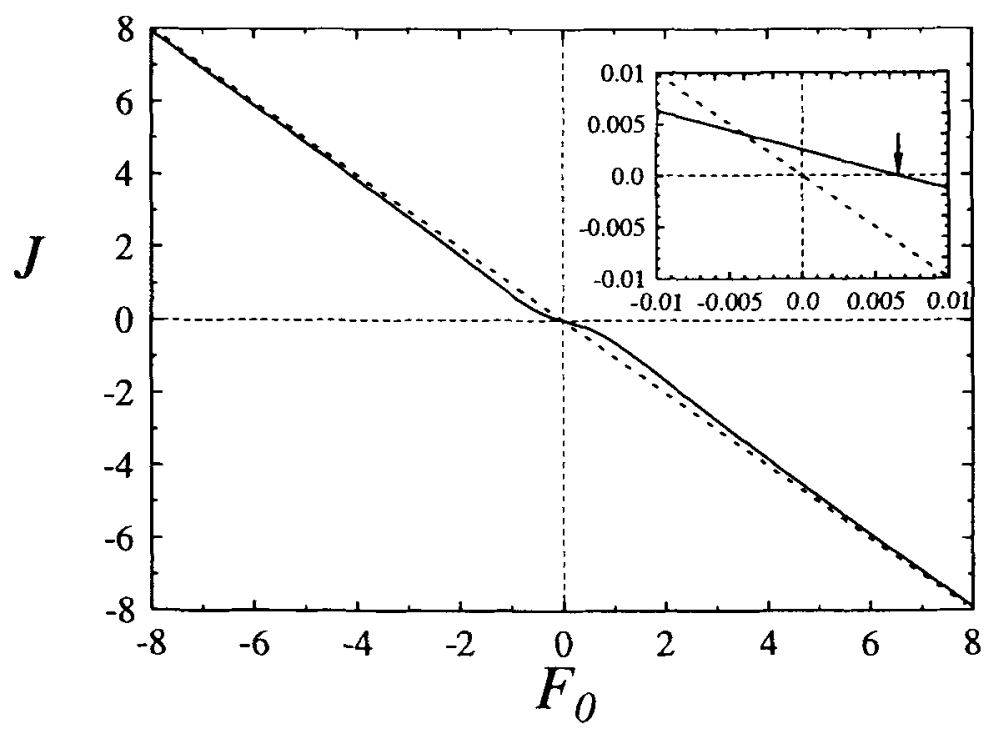

Fig. 8. Current versus load characteristics (solid line) for the correlation ratchet in eqs. (10),(11). The probability current $J$ is evaluated for the parameters: White noise intensity $D=0.1$, colored noise intensity $Q=0.025$, noise correlation time $\tau=0.25$, and the ratchet potential $V_{R}$ used in Fig. 3 . The inset gives a magnification of the current-load curve within the linear response regime of small $F_{0}$. Note the two different linear behaviors at small and large load forces $F_{0}$. In the regime of positive load forces $0 \leq F_{0} \leq F_{\text {stop }}$ with $F_{\text {st op }}=0.0066$ denoting the stopping force (see the arrow in the inset), the particles move uphill in the tilted potential $V_{R}(x)+x F_{0}$. For comparison, we indicate by the dotted line the diagonal $J=-F_{0}$.

With the stopping force $F_{\text {stop }}$ being proportional to the no-load current, the behavior of $F_{\text {stop }}(\tau)$ versus noise color $\tau$ mimics the same behavior that 
characterizes the current versus $\tau$. For different noise scalings, e.g., constant intensity noise versus constant variance noise, i.e., $Q \rightarrow Q \tau$ [26], the behavior of the stopping force changes accordingly, see in [26]. For colored noise $\zeta(t)$ with constant intensity the stopping force $F_{\text {stop }}(\tau)$ exhibits a characteristic resonance-like behavior; for constant variance noise, $F_{\text {stop }}(\tau)$ versus $\tau$ is a monotonically increasing function.

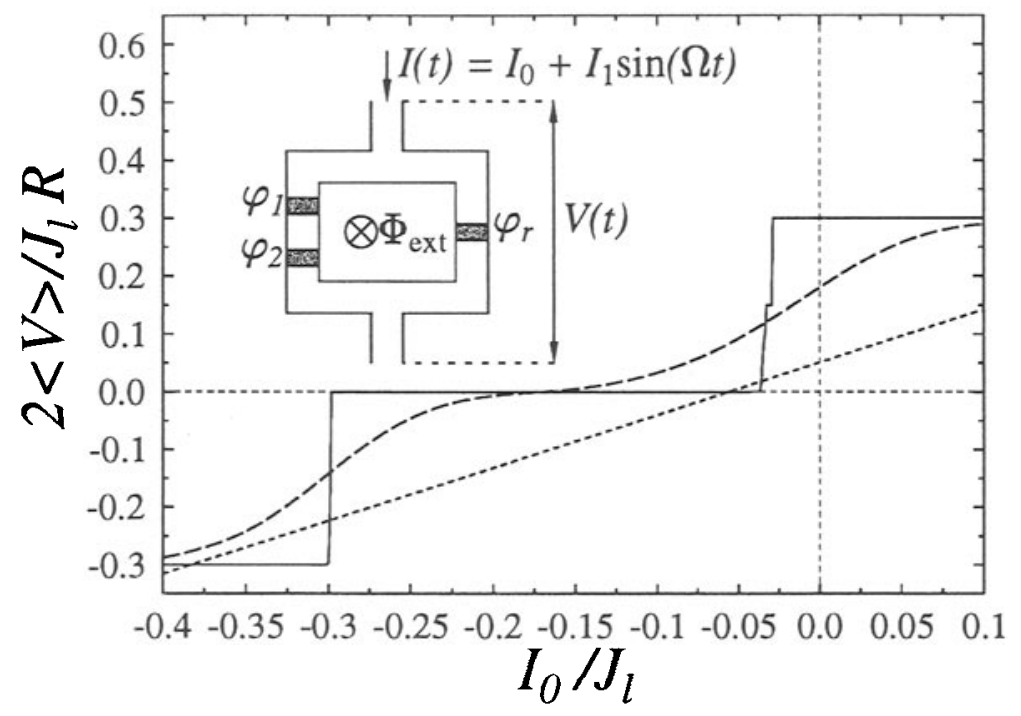

Fig. 9. Voltage rectification in a SQUID ratchet [30]: The schematic picture depicts the SQUID arrangement composed of three Josephson junctions with corresponding Josephson phases $\varphi$ that is threaded by an external flux $\Phi_{\text {ext }}$. $J_{l}$ denotes the critical current of one of the two identical Josephson junctions in the left arm and $R$ is its resistance. The average voltage $\langle V\rangle$ across the right Josephson junction is depicted versus the dc-component $I_{0}$ of the current source $I(t)$. The ac-component $I_{1}$ of the rocking SQUID is driven with frequency $\Omega=0.3$ and strength $A=I_{1} / J_{l}=1.7$. The white (Nyquist) noise intensity is $D=0$ (solid), 0.01 (dashed), and 0.5 (dotted). With this scaling the SQUID ratchet is modeled by (2) and the ratchet potential of Fig. 3, but with a spatial period of $L=2 \pi$, instead of $L=1$ in Fig. 3, and complemented by a static load of $F_{0}=I_{0} / J_{l}$ [this corresponds to a negative tilt $-x F_{0}$ of $\left.V_{R}(x)\right]$. Note that the voltage-current characteristics exhibits a range of parameters in which a fixed sign (in a narrow regime around zero even a fixed value) of the voltage occurs, regardless of the sign of the load $I_{0}$.

The rocking ratchet mechanism is particular interesting for novel technological applications. With the mechanism working even in absence of noise it 
can be used not only on mesoscopic, micro- and nanoscales but even can extend from a pure quantum noise dominated regime to macroscopic scales with a strict deterministic dynamical behavior. This yields novel separation techniques for particles on all scales. As mentioned previously, surfaces such as blazed gratings which are subjected to alternating electric fields [14], as well as gratings induced by optical radiation forces [15] can be used to separate monodisperse, microsized particles. A particular noteworthy ratchet application is the current-voltage rectification in superconducting quantum interference devices (SQUID). With an asymmetric SQUID (see inset in Fig. 9) driven by an ac-current we find [30] that there exists a range of parameters in which a fixed sign (in a narrower regime even a fixed value) of the average voltage occurs, independent of the sign of the external dc-current source, cf. Fig. 9. Hence this system presents another case in which novel physics results due to the ratchet effect.

The authors acknowledge support by the Deutsche Forschungsgemeinschaft (Az. Ha 1517/13-1). We also like to thank our co-authors P. Jung, J. Kissner, J. Luczka, P. Reimann, and P. Talkner for many insightful discussions. We also profited from suggestive discussions with $\mathrm{Ch}$. Doering, L. Schimansky-Geier, and M. Bier.

\section{References}

[1] R. P. Feynman, R. B. Leighton, and M. Sands, The Feynman Lectures on Physics (Addison Wesley, Reading, MA, 1966), Vol. 1, chapt. 46.

[2] M. von Smoluchowski, Phys. Z. XIII, 1069 (1912).

[3] S. Leibler, Nature 370, 412 (1994); J. Maddox, ibid. 365, 203 (1993); J. Maddox, ibid. 369, 181 (1994); J. Maddox, ibid. 368, 287 (1994).

[4] R. Landauer, Phys. Rev. A 12, 636 (1975).

[5] M. Büttiker, Z. Phys. B 68, 161 (1987); N. G. Van Kampen, IBM J. Res. Devel. 32, 107 (1988); R. Landauer, J. Stat. Phys. 53, 233 (1988).

[6] R. L. Stratonovich, Theory of Random Noise (Gordon and Breach, New York, 1967), Vol. 2, chap. 9; H. Risken, The Fokker-Planck Equation (Springer, Berlin, 1984).

[7] P. Reimann, Current reversal in a white noise driven flashing ratchet, preprint (1996).

[8] J. M. R. Parrondo and P. Espanol, Criticism of Fynman's analysis of the ratchet as an engine, American J. Phys. (1996), in press.

[9] A. Ajdari, Ph.D. thesis, Universite Paris, 1992, chap. 7. A. Ajdari and J. Prost, C. R. Acad. Sci. Paris 315, 1635 (1992); J. Prost, J.-F. Chauwin, L. Peliti, and A. Ajdari, Phys. Rev. Lett. 72, 2652 (1994); J. F. Chauwin, A. Ajdari, and J. Prost, Europhys. Lett. 27, 421 (1994); J. F. Chauwin, A. Ajdari, and J. Prost, Europhys. Lett. 32, 373 (1995).

[10] R. D. Astumian and M. Bier, Phys. Rev. Lett. 72, 1766 (1994).

[11] A. Mielke, Ann. Physik 4, 721 (1995).

[12] T. V. Elston and C. R. Doering, J. Stat. Phys. 83, 359 (1996). 
[13] P. Hänggi, Z. Phys. B 31, 407 (1978); C. Van den Broeck and P. Hänggi, Phys. Rev. A 30, 2730 (1984).

[14] J. Rousselet, L. Salome, A. Ajdari, and J. Prost, Nature 370, 446 (1994).

[15] L. P. Faucheux, L. S. Bourelieu, P. D. Kaplan, and A. J. Libchaber, Phys. Rev. Lett. 74, 1504 (1995).

[16] M. O. Magnasco, Phys. Rev. Lett. 71, 1477 (1993).

[17] A. Ajdari, D. Mukamel, L. Peliti, and J. Prost, J. Phys. I (France) 4, 1551 (1994).

[18] R. Bartussek, P. Hänggi, and J. G. Kissner, Europhys. Lett. 28, 459 (1994).

[19] P. Talkner, R. Badii, R. Bartussek, and P. Hänggi, Stroboscopic description of rocking ratchets, preprint (1996).

[20] P. Jung, J. G. Kissner, and P. Hänggi, Phys. Rev. Lett. 76, 3436 (1996).

[21] P. Reimann, R. Bartussek, R. Häußler, and P. Hänggi, Brownian Motors Driven by Temperature Oscillations, Phys. Lett. A (1996), in press.

[22] C. R. Doering, W. Horsthemke, and J. Riordan, Phys. Rev. Lett. 72, 2984 (1994).

[23] M. M. Millonas and M. I. Dykman, Phys. Lett. A 185, 65 (1994).

[24] A. Mielke, Ann. Physik 4, 476 (1995).

[25] J. Luczka, R. Bartussek, and P. Hänggi, Europhys. Lett. 31, 431 (1995).

[26] R. Bartussek, P. Reimann, and P. Hänggi, Phys. Rev. Lett. 76, 1166 (1996).

[27] J. Kula, T. Czernik, and J. Luczka, Phys. Lett. A (1996), in press.

[28] M. Bier, Phys. Lett. A 211, 12 (1996).

[29] I. Derényi and T. Vicsek, Phys. Rev. Lett. 75, 374 (1995).

[30] I. Zapata, R. Bartussek, F. Sols, and P. Hänggi, Voltage rectification by a SQUID ratchet, submitted to Phys. Rev. Lett. (1996). 